EPJ Web of Conferences 87, 03003 (2015)

DOI: $10.1051 /$ epjconf/20158703003

(C) Owned by the authors, published by EDP Sciences, 2015

\title{
New approach to ECE measurements based on Hilbert-transform spectral analysis
}

\author{
Hitesh Kumar B. Pandya ${ }^{1}$ and Yuriy Divin ${ }^{2}$ \\ ${ }^{1}$ ITER-India, Institute for Plasma Research, Gandhinagar-380025, India; hitesh@ipr.res.in \\ ${ }^{2}$ Peter Grünberg Institute, Forschungszentrum Jülich, 52425 Jülich, Germany ; Y.Divin@,fz-juelich.de
}

\begin{abstract}
Spectroscopy of Electron Cyclotron Emission (ECE) has been established as adequate diagnostic technique for fusion research machines. Among various instruments for ECE diagnostics, only Fourier-transform spectrometers with Martin-Puplett interferometers can measure electron cyclotron radiation in a broadband frequency range from 70 to $1000 \mathrm{GHz}$. Before these measurements, a complete system including a frontend radiation collector, a transmission line, an interferometer and a radiation detector should be absolutely calibrated. A hot/cold calibration source and data-averaging technique are used to calibrate the total ECE diagnostic system. It takes long time to calibrate the ECE system because of the low power level of the calibration source and high values of the noise equivalent power (NEP) of the detection system. A new technique, Hilbert-transform spectral analysis, is proposed for the ITER plasma ECE spectral measurements. An operation principle, characteristics and advantages of the corresponding Hilbert-transform spectrum analyser (HTSA) based on a high- $T_{c}$ Josephson detector are discussed. Due to lower NEP-values of the Josephson detector, this spectrum analyser might demonstrate shorter calibration times than that for the Martin-Puplett interferometer.
\end{abstract}

\section{Introduction}

The measurement of the cyclotron radiation provides the plasma electron temperature profile and also used to study various phenomena in plasma physics like temperature fluctuations, non-thermal electrons population and power losses due to ECE. The ECE measurement diagnostic has been established as adequate diagnostic technique for fusion research machines. Therefore, measurement of this diagnostic is proposed for the ITER plasma.

There are many techniques for the measurement of the cyclotron radiation.
Among them, superheterodyne radiometers and Martin-Puplett interferometers are very popular and used for ECE measurements in a majority of fusion research machines [1]. The radiometers have a limited frequency bandwidth, determined by an intermediate frequency (IF) band width and a frequency of local oscillators, e.g. from 100 to $200 \mathrm{GHz}$. A combination of an optical-mechanical Fouriertransform spectrometer, based on a polarizingtype Martin-Puplett interferometer, with a broadband cryogenic detector is used for broadband ECE measurements. Here, the usual cryogenic detector is an InSb hotelectron bolometer, which operates in an optical cryostat at the temperature of $4.2 \mathrm{~K}$. 
This technique has been used for broadband ECE measurements for around 40 years.

The required specifications of the spectrometer for the ECE measurement are mentioned in Table 1. The ECE total system including a front-end radiation collector, a transmission line, an interferometer and a radiation detector should be absolutely calibrated. A hot/cold calibration source and data-averaging technique are used to calibrate the total ECE diagnostic system. It takes long time to calibrate the ECE system because of the low power level of the calibration source and high values of the noise equivalent power $(N E P)$ of the detection system. There are two ways to reduce the calibration time for the ECE measurement system. One is to improve the transmission efficiency of the ECE transmission line and other is to have a broad band ECE measurement instrument with low NEP detection system. We propose a new type of a $\mathrm{THz}$ spectrum analyzer, based on a high$T_{c}$ superconducting Josephson junction with low $N E P$-values [2] for the ITER plasma ECE spectral measurements.

This new technique is Hilbert-transform spectral analysis where a high- $T_{c}$ superconducting Josephson junction is used as a detector. An operation principle, characteristics and advantages of the corresponding Hilbert-transform spectrum analyzer (HTSA) based on the high- $T_{c}$ Josephson detector are discussed in this paper. Due to lower $N E P$-values of the Josephson detector, this spectrum analyzer might demonstrate shorter calibration times than that for the Martin-Puplett Interferometer. This calculated result is also presented in this paper. More details of the HTSA measurement proposal for the ITER ECE measurement is given elsewhere [3].

Table 1

\begin{tabular}{|l|l|}
\hline Spectral range $(\mathrm{GHz})$ & $70-1000$ \\
\hline Frequency resolution $(\mathrm{GHz})$ & $7.5-10$ \\
\hline Scanning repetition rate $(\mathrm{ms})$ & $10-20$ \\
\hline $\begin{array}{l}\text { Detector System Optical Responsivity } \\
(\mathrm{V} / \mathrm{W})\end{array}$ & $\geq 2,000$ \\
\hline $\begin{array}{l}\text { Detector System Optical Noise } \\
\text { Equivalent Power (W/Hz }\end{array}$ & $\leq 1.2 \times 10^{-12}$ \\
\hline Detector dynamic range $(\mathrm{dB})$ & $\geq 29 \mathrm{~dB}$ \\
\hline Duty cycle & Continuous \\
\hline
\end{tabular}

\section{HTSA Theory}

There is a modification of the dc $I(V)$ curve of the Josephson junction due to frequency pulling of Josephson oscillations induced by external radiation. The small-signal response $\Delta I(V)=I(V)-I_{0}(V)$ has been found to be proportional to the Hilbert transform of the spectral density $S(f)$ of external radiation, so unique deconvolution of $S(f)$ is possible from the experimental data [4]. The exact solution of the problem is

$S(f)=\left(\frac{1}{\pi}\right) \cdot \mathrm{P} \int_{-\infty}^{\infty} \frac{H\left(f_{j}\right)}{\left(f-f_{j}\right)} d f$,

where $f_{j}=2 \mathrm{e} V / \mathrm{h}$ is the voltage-controlled frequency of the Josephson oscillations, $V$ is the voltage across the junction, $H(V)$ is a normalized response function

$H(V)=(8 / \pi) \cdot(\mathrm{h} / 2 \mathrm{e}) \cdot\left[\Delta I(V) \cdot I_{0}(V) \cdot V / I_{c}^{2} R_{n}^{2}\right]$,

$I_{c}$ is the critical current and $R_{n}$ is the normal state resistance of the Josephson junction, $f$ is the radiation frequency, $h$ and $e$ are the Planck's constant and the electron charge, respectively. This relation between spectrum and the response function is valid when the Josephson junction is described by the resistively shunted junction (RSJ) model [5]. The radiation spectrum can be recovered in three steps: one should measure the $I-V$ curves of the junction with and without radiation, calculate the normalized response function $H(V)$ from experimental data according to equation (2) and then apply the Hilbert transform to the function $\mathrm{H}(\mathrm{V})$ according to equation (1).

There is an additional advantage in the Hilbert transform that filters the background radiation, because the Hilbert transform gives a zero value when applied to the constant spectral density. 


\section{Characteristics of HTSA}

In this section we give a description of various features of HTSA, based on high- $T_{c}$ Josephson junctions. To derive the required parameters of the HTSA for ECE applications, the following set of parameters of Josephson junctions will be considered: the junction temperature $T=66 \mathrm{~K}$, the normal state resistance $R_{n}=0.75 \mathrm{Ohm}$, the characteristic power level $P_{0}(66 \mathrm{~K})=6.4 \mu \mathrm{W}$ and the temperature-dependent characteristic Josephson voltage $I_{c}(T) R_{n}=0.11 \mathrm{mV}$ at $66 \mathrm{~K}$.

\subsection{Spectral range}

The spectral bandwidth of HTSA is actually the spectral range of Josephson oscillations that is within two main frequency limits. The low frequency limit $f_{l}$ is given by

$$
f_{l}=\left(1.5 \delta f_{0}(T) f_{c}^{2}(T)\right)^{1 / 3}
$$

where $\delta f_{0}$ is thermal broadening of the Josephson line width at large voltages and given by

$$
\delta f_{0}=4 \pi(2 e / h)^{2} k T R_{n}
$$

The high-frequency limit $f_{h}$ is given by

$$
f_{h}=2 e\left(P_{0} R_{n}\right)^{1 / 2} / h,
$$

where $P_{0}(T)$ is the characteristic power level of Joule heating in the junction.

In high- $T_{c}$ Josephson junctions, the ac Josephson effect has been demonstrated in the range from $5 \mathrm{GHz}$ to $5 \mathrm{THz}$ [6] by combining the values of temperature $T$, characteristic voltage $I_{c}(0) R_{n}$ and $R_{n}$. For predetermined parameters of the Josephson junction, the characteristic Josephson frequency $f_{c}$ is 53 $\mathrm{GHz}$ and the Josephson line width $\delta f_{0}(T)$ is 2 $\mathrm{GHz}$ from equation (4). The low-frequency limit $f_{l}$ is $20 \mathrm{GHz}$ and the high-frequency limit $f_{h}$ is $1060 \mathrm{GHz}$ for the same set of parameters. So, the required spectral range from 70 to $1000 \mathrm{GHz}$ given in Table 1 will be within the frequency limits of the HTSA base on the high- $T_{c}$ Josephson junction with predetermined parameters.

\subsection{Frequency Resolution}

A frequency resolution of the Josephson frequency selective detection and Hilbert transform spectral analysis is determined by the line width $\delta f$ of Josephson radiation of the junction. An instrumental function of Hilbert transform spectroscopy is determined by spectrum of Josephson oscillation, which is described by Lorentz function for broadband thermal voltage fluctuations. The Josephson linewidth is given by [6]

$$
\delta f=\delta f_{0}\left(2 \omega^{2}+3\right) / 2 \omega^{2}
$$

where $\omega$ is the normalized voltage or frequency, $\omega=V / I_{c} R_{n}=f_{j} / f_{c}$ and $f_{j}$ is the measurement frequency. The frequency resolution of the spectrometer decreases with the measurement frequency $f j$ and for abovegiven values of junction parameters, the variation in frequency resolution from 3.7 $\mathrm{GHz}$ to $2 \mathrm{GHz}$ is presented in Fig. 1.

\subsection{Time Resolution}

Intrinsic time constants of high- $T_{c}$ Josephson junctions is quite low, less than $10^{-9} \mathrm{~s}$. The time resolution of the spectrum analyser is determined by a bandwidth of available lownoise electronics. Electronics with the bandwidth of $14 \mathrm{MHz}$ and noise of $1.6 \times 10^{-10}$ $\mathrm{V} / \mathrm{Hz}^{1 / 2}$ have already been realized for operation with high- $\mathrm{T}_{\mathrm{c}}$ Josephson junctions that gives good time resolution of 71 ns. Therefore, we can achieve required time resolution of less than $10 \mathrm{~ms}$ for the ECE application. 


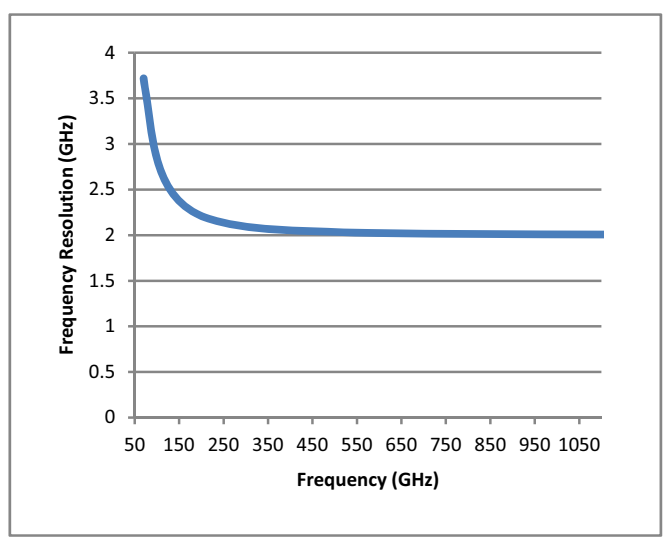

Figure 1 The variation of the frequency resolution of the spectrum analyzer from 70 to $1000 \mathrm{GHz}$.

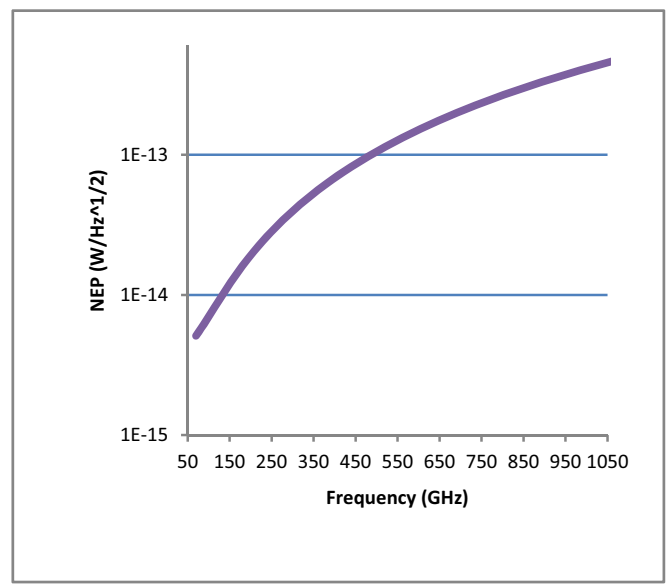

Figure 2 NEP vs. frequency inside frequency range 70 to $1000 \mathrm{GHz}$

\subsection{Noise Equivalent Power (NEP) and Dynamic Range}

Intrinsic $N E P$ of the spectrum analyser is described by the following equation [7]

$$
\begin{gathered}
N E P= \\
2^{1 / 2} 8 \pi(2 e / h)(k T)^{3 / 2} R_{n}^{1 / 2}\left(\left(3+2 \omega^{2}\right)^{3 / 2} / \omega\right)
\end{gathered}
$$

This can be improved by operating with low resistance junctions and at lower temperatures. The NEP-values are also dependent on the measurement frequency (see Fig. 2).

A power dynamic range $\mathrm{D}$ is determined by the ratio of $P_{m} / N E P^{*} B^{1 / 2}$, where $\mathrm{P}_{\mathrm{m}}$ is the maximum power, where response $\Delta I(P)$ of the detector is deviating from the linear dependence on the fixed amount, e.g. $3 \mathrm{~dB}$, $N E P$ is the noise equivalent power and $B$ is the bandwidth of the electronics of the detector. The dynamic range $D$ of the frequency selective Josephson detector is described by the following equation [8]

$$
\begin{aligned}
& D= \\
& 10 \pi / 2^{1 / 2}(2 e / h)\left(k T R_{n}\right)^{1 / 2}\left(\left(3+2 \omega^{2}\right)^{1 / 2} / \omega\right)
\end{aligned}
$$

The experimental response $\Delta I(V)$ to $86 \mathrm{GHz}$ radiation is presented in Reference 11 . The calculated dynamic range for our parameters is plotted in Figure 3.

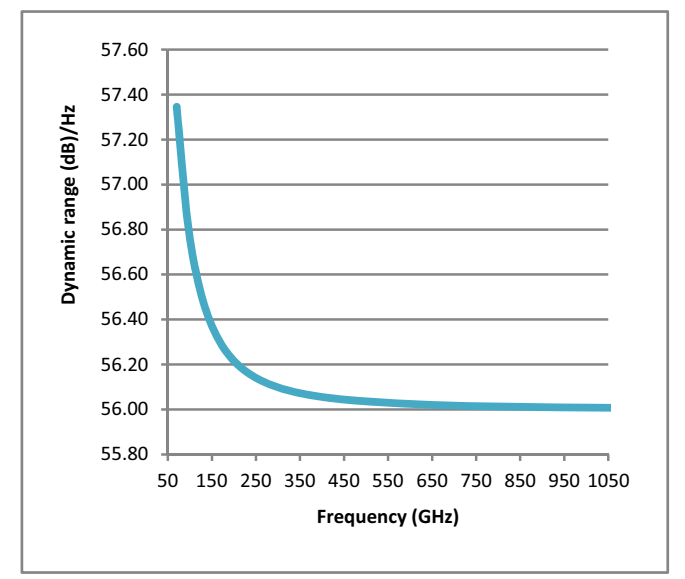

Figure 3 Variation of power dynamic range with frequency 


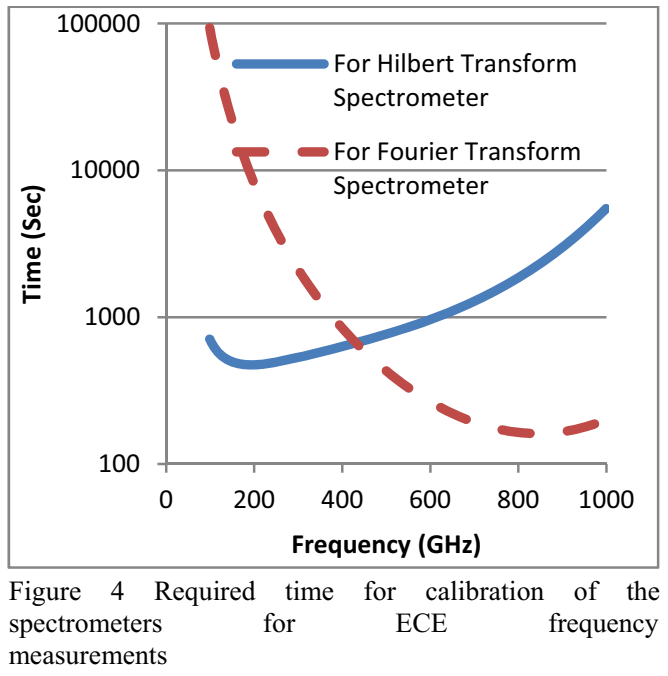

\section{Spectrometer Calibration and required calibration time}

It is required to calibrate the ECE radiation measurement instrument for determining the radiation temeprature. This calibration can be done by placing the calibration radiation

source at the front end (near tokamak) of the ECE measurement system. A hot/cold black body source with two known physical temperatures is used in a calibration process. The hot source consists of a silicon carbide plate with surface contoured to assure that it is a blackbody emitter while the cold source is based on Eccosorb absorbers cooled by liquid nitrogen. This calibration method with two different radiation temperatures gives reasonably good accuracy of $10 \%$ for temperature measurements.

The NEP of the spectrometer is higher than the power level of the signal from the calibration source. Therefore, a data averaging methode is used to calibrate the spectrometer. When $n$ numbers of measurement data are averaged, the improvement in signal/noise ratio is equal to $n^{0.5}$. The required calibration time depends on the NEP of the spectrometer and the power level of the signal. For the signal to noise ratio of 100 , the required total calibration time is given by

$$
T_{i}=10000\left(\frac{N E P}{P_{S}}\right)^{2} B_{v} \Delta \tau,
$$

where $P_{s}$ is the signal power, $B_{v}$ is the video bandwidth and $\Delta \tau$ is time taken to scan full spectrum. The signal power $P_{s}$ during calibration is given by

$$
P_{s}=K \theta_{s} f^{2} \Delta f E_{t} T_{t l},
$$

where $\theta_{s}$ is the calibration source temperature, $f$ is the frequency of radiation, $\Delta f$ is the frequency resolution of the spectrometer, $E_{t}$ is the etendue, $T_{t l}$ is the power transmission from the source to the detector and the constant $K$ is equal to $2.44 \times 10^{-41}$.

The estimated calibration time for ECE measurements vs. frequency for both types of spectrometer is shown in Fig. 4 for the following values of parameters: $\theta_{s}=973 \mathrm{~K}$, $\Delta f=3.75 \mathrm{GHz}, E_{t}=1.28 \times 10^{-5} \mathrm{~m}^{2} \mathrm{Sr}$. We have considered the power transmission $\left(T_{t l}\right)$ of the circular waveguide transmission line [9] for this calculation. The video bandwidth $B_{v}$ is $100 \mathrm{KHz}$ and the time taken to scan full spectrum is $10 \mathrm{~ms}$. The required calibration time for Hilbert-transform spectral analyser is of one-two orders of magnitude less than that for Martin-Puplett-type Fourier-transform spectrometer at lower frequency range up to $350 \mathrm{GHz}$. This lower frequency range shall be used to determine a radial profile of the plasma electron temperature [9].

\section{Conclusions}

The theoretical estimates shown above demonstrate full compliance of main characteristics of the Hilbert-transform spectrum analyser based on the high- $T_{c}$ Josephson junction with the required specifications for measurements of ECE radiation spectrum and radiation temperatures in fusion research devices. It is inferred that the required calibration time for the Hilberttransform analyzer is less than that for MartinPuplett-type Fourier-transform spectrometer.

Additional advantage of the Hilbert-transform spectrum analyzer in ECE measurements comes from the principal difference between 
Hilbert-transform and Fourier-transform techniques in detection of radiation with spectra consisting of a spectral line and a frequency-independent background. A frequency-independent radiation background gives no signal in Hilbert-transform spectral analysis, but it gives a large signal in Fouriertransform spectroscopy. An effective power dynamic range available for ECE peaks embedded in background radiation might be higher in Hilbert-transform spectral analysis compared with that for Fourier-transform spectroscopy.

Also, when compared with the Fouriertransform spectrometer for ECE measurements, the Hilbert-transform spectrum analyzer contains no moving mechanical parts and does not require cooling to liquid helium temperatures.

\section{References}

1. A. E. Costley, Proc. Course and Workshop on Basic and Advanced Diagnostic Technique for Fusion Plasma 119 (1986)

2. Y. Divin, M. Lyatti, A. Snezhko, U. Poppe, and V. Pavlovskiy, IEEE Trans. Appl. Supercond., 23, 1800204 ( 2013)

3. Y. Divin and Hitesh Kumar B. Pandya, Fusion Science \& Technology, 2014 (to be published)

4. Yu.Ya. Divin, O.Yu. Polyanski, A.Ya. Schul'man. , IEEE Trans. Magn., 19, 613 (1983)

5. K. K. Likharev: Dynamics of Josephson Junctions and Circuits (Gordon and Breach, New York 1986) pp. 302, 303, 304, 309.

6. Y. Divin, U. Poppe, V.N. Gubankov, K. Urban IEEE Sensors J., 8, 750 (2008)

7. V. V. Shirotov and Y. Y. Divin, Techn. Phys. Lett., 30, 522 (2004)

8. M. Lyatti, Y. Divin, O. Volkov, V. Pavlovskii, V. Gubankov, K. Urban IEEE Trans. Appl. Supercond., 17, 332 (2007)

9. Hitesh Kumar B. Pandya, V.S. Udintsev, G. Vayakis and Max Austin, PP. 03005-1 in proceeding of $17^{\text {th }}$ Workshop on ECE and ECRH at Deurne, The Netherlands, 7 - 10 May, 2012 